Revue des patrimoines

$20 \mid 2013$

Les patrimoines de la traite négrière et de l'esclavage

\title{
Patrimoine et mémoire de l'esclavage en Haïti : les vestiges de la société d'habitation coloniale
}

Jacques de Cauna

\section{(2) OpenEdition}

Journals

Édition électronique

URL : http://journals.openedition.org/insitu/10107

DOI : 10.4000/insitu. 10107

ISSN : 1630-7305

Éditeur

Ministère de la Culture

Référence électronique

Jacques de Cauna, « Patrimoine et mémoire de l'esclavage en Haïti : les vestiges de la société d'habitation coloniale », In Situ [En ligne], 20 | 2013, mis en ligne le 30 mai 2013, consulté le 10 décembre 2020. URL : http://journals.openedition.org/insitu/10107 ; DOI : https://doi.org/10.4000/ insitu. 10107

Ce document a été généré automatiquement le 10 décembre 2020.

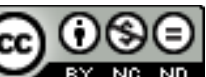

In Situ Revues des patrimoines est mis à disposition selon les termes de la licence Creative Commons Attribution - Pas d'Utilisation Commerciale - Pas de Modification 4.0 International. 


\title{
Patrimoine et mémoire de l'esclavage en Haitt : les vestiges de la société d'habitation coloniale
}

\author{
Jacques de Cauna
}

Les sucreries sont la sueur et le sang de nos aïeux :

il faut les respecter

Les étudiants de l'École Nationale des Arts, Frontispice de l'exposition Les Sucreries, Institut Français d'Haïti, 1989.

1 La première république noire du monde est aujourd'hui encore un véritable conservatoire $\mathrm{du}$ patrimoine historique de l'économie esclavagiste du XVIII ${ }^{\mathrm{e}}$ siècle dont la grande plantation - connue sous le nom d'habitation aux Isles d'Amérique - était l'unité de base ${ }^{1}$. Comme dans l'ensemble des sociétés créoles antillaises, qu'elles soient francophones, anglophones, ou hispanophones, la grande plantation fut, en effet, le cadre de vie, de mort et de travail quotidien de la majorité des esclaves - nègres à talents, le plus souvent créoles, ou nègres de houe, en grande majorité bossales. Elle a perduré longtemps après les abolitions de l'esclavage.

2 Entre 1975 et 1990, à partir de repérages effectués dans des documents d'archives des $\mathrm{XVIII}^{\mathrm{e}}$ et XIX ${ }^{\mathrm{e}}$ siècles suivis d'explorations et d'enquêtes d'archéologie industrielle de terrain, plus de 2500 clichés de vestiges de la société d'habitation coloniale esclavagiste de Saint-Domingue et de son environnement urbain, militaire, religieux ou naturel, ont été pris en Haïti afin de garder trace d'un patrimoine menacé.

Ces clichés concernent principalement les caféteries du front pionnier des mornes (plus de 3000 à Saint-Domingue, occupant $60 \%$ des terres cultivées), les indigoteries (autant, souvent associées aux cotonneries), guildiveries (qui fabriquaient le tafia), chaufourneries ou briqueteries, et surtout les sucreries ( $40 \%$ de toute la fortune coloniale avec 900 unités de production sur seulement $14 \%$ des terres), sources de la plus grande richesse avec des ateliers de 2 à 300 esclaves et un investissement en matériel élevé dans un cadre dont les 
plans, gravures et inventaires d'époque, et surtout les vestiges actuels disséminés dans les campagnes haïtiennes, révèlent l'organisation immuable entre bâtiments d'exploitation et d'habitation des grandes sucreries : au vent, au bout de la grande allée ouverte par un portail monumental à deux ou quatre piliers et grille en fer forgé, la Grand-case [maison de maître] en position dominante dans son enclos, avec ses annexes et dépendances (cuisine, poulailler, jardin, entrepôts, remises, cases des domestiques...) ; au-devant, la savane (ou «la cour») où paissent les bêtes; plus loin, pour éviter aux maîtres bruits, odeurs et risques d'incendie, les installations industrielles (aqueducs, moulins, sucreries, purgeries, étuves...) ; puis le quartier des esclaves, sous le vent ; le tout entouré de terres réservées aux plantations de denrées exportables et de vivres alimentaires pour l'atelier (bananes, manioc, riz, patates...).

4 Cinq cents de ces clichés ont été sélectionnés et positionnés sur la carte de l'Isle de SaintDomingue Partie Françoise ou Hayti, 1789, revue et corrigée en 1804, par Delvaux, afin que l'on puisse localiser chaque site et chaque ruine de ces habitations qui ont produit la plus grande richesse coloniale de l'époque moderne.

5 En dehors des vestiges historiques urbains ou militaires, les ruines des quelque 8500 habitations coloniales - le plus important réseau d'exploitation des Antilles constituent actuellement un patrimoine d'une exceptionnelle richesse qui nécessite protection car il est très menacé et fragilisé par les déprédations climatiques et humaines.

Ces clichés témoignent de l'histoire des plantations esclavagistes, mais aussi, comptetenu de la rapide disparition depuis quelques années de ces bâtiments, ils constituent un sauvetage virtuel de ce patrimoine historique et mémoriel.

\section{Aperçus sur le système des habitations et l'empreinte coloniale en Haïti}

7 La grande plantation, connue sous le nom d'habitation aux Isles d'Amérique françaises, est le véritable creuset de la société créole antillaise : unité de base de la production pour les économistes, elle est aussi pour les anthropologues le premier lieu de mutation interraciale où s'est forgée l'identité socio-culturelle spécifique des Iles, principalement avec l'introduction de l'industrie sucrière à partir du milieu du XVII ${ }^{e}$ siècle et son inévitable corollaire, l'esclavage des Noirs. Alors que le premier mouvement d'implantation des Français aux Iles, popularisé par l'épopée des Frères de la Côte, n'a laissé que peu de traces originales, la colonisation agricole liée à l'apparition des grands domaines a imprégné de sa marque indélébile et modelé en profondeur le nouveau monde ainsi créé. Ainsi dans un pays comme l'actuelle république (l'ancienne Saint-Domingue) qui est resté essentiellement rural, l'habitation est encore aujourd'hui la première référence, la plus petite unité pratique de vie et de reconnaissance pratiques, l'objet de la première question que posera le paysan haïtien à celui qu'il ne connaît pas encore : ki bitation nou soti? [« de quelle habitation es-tu?»]. Le phénomène, certes, n'est pas exclusivement français et a ses homologues brésiliens, hispaniques ou anglais ${ }^{2}$, mais il présente encore aujourd'hui aux îles françaises une remarquable originalité qui les distingue encore plus nettement que leurs voisines de leur environnement proche.

Cette société d'habitation, création locale originale, s'est mise en place, progressivement mais inexorablement, entre les aventures individuelles désordonnées des premiers temps de la colonisation (la flibuste) et les débris des tentatives de peuplement officielles (le 
système des engagés blancs voué à l'échec par le peu de cas fait des malheureux Trente-six mois $^{3}$ ) sans lendemains car coupés du système de colonisation agricole sous contrôle métropolitain qui allait tout emporter ${ }^{4}$. À son apogée, à la veille de la Révolution, elle est marquée par la large prédominance à Saint-Domingue - dans la proportion de plus de $40 \%$ - de colons gascons et aquitains venus par le port de Bordeaux. Ce sont eux qui y laissent la marque la plus forte dont les traces les plus évidentes apparaissent très clairement dans des modèles architecturaux tels que les portails d'habitations semblables à ceux des vignobles bordelais et cognaçais ou l'architecture de grand-cases aux allures de chartreuses bordelaises, les formes de toits et leur ornementation, les corniches, galeries, colombiers, cours et puits, et, dans les villes, le quadrillage des rues rappelant les bastides gasconnes, mais aussi dans la micro-toponymie locale qui a conservé assez fidèlement pour la plupart des noms de lieux, ceux de leurs anciens propriétaires coloniaux (Lalanne, Carrère, Laborde, Marsan, Navailles, Lafaurie, Dupoey, Nolivos...), les noms de familles (Gardères, Castéra, Dupuy, Sansaricq, Casamajor, les présidents Salnave, Dartiguenave...), ou encore dans un ensemble de mœurs et de coutumes remarquablement vivaces alliées au sentiment d'une différence marquée au sein d'une Amérique anglophone et hispanophone, et jusqu'à des manières de parler (prononciation accentuée des finales), d'être ou de paraître qui ont profondément marqué les mentalités.

Il faut rappeler, par ailleurs, que l'ancienne Saint-Domingue - véritable Far West colonial français - était à la fin du XVIII ${ }^{\mathrm{e}}$ siècle, et de très loin, la plus grande $\left(27000 \mathrm{~km}^{2}\right)$, la plus peuplée (au moins 600000 habitants dont 30000 Blancs) et la plus riche ( 7 fois plus que la Martinique ou la Guadeloupe) des îles à sucre antillaises de la Caraïbe conservées à La France par le traité de Paris au détriment des « quelques arpents de neige du Canada ». La "Reine des Antilles... orgueil de la France dans le Nouveau-Monde", selon son historiographe Moreau de Saint-Méry, - qui s'étendait sur un territoire vingt-cinq fois plus grand que celui de la Martinique par exemple avec une population six fois supérieure - était devenue à l'époque le lieu de destination d'au moins huit passagers sur dix au départ pour les îles, produisant à elle seule six à sept fois plus que chacune des petites Antilles françaises, davantage même que toutes les Antilles anglaises et espagnoles réunies, et fournissant, avec les réexportations, près des deux-tiers du commerce extérieur de la France, et notamment les $3 / 4$ du sucre et les $2 / 3$ du café mondiaux. Étudier Saint-Domingue à travers la contribution de la région qui a fourni le plus grand nombre de colons, c'est donc saisir l'essentiel des réalités antillaises de l'époque ${ }^{5}$.

Premier établissement de Christophe Colomb, la colonie avait été rapidement dépeuplée de ses habitants indiens pour devenir terre d'élection des flibustiers et boucaniers dans sa partie occidentale. Son prodigieux essor datait de la seconde moitié du XVIII ${ }^{e}$ siècle avec le développement de l'économie agro-industrielle de plantation (sucre, café, coton, indigo...) due à l'exploitation massive d'esclaves noirs jusque dans les années 1790 . Les troubles révolutionnaires dans la métropole coloniale et au niveau local déstabilisèrent l'ordre esclavagiste et entraînèrent la perte de la colonie par la France en une quinzaine d'années de luttes, de 1791 à son indépendance, proclamée le $1^{\text {er }}$ janvier 1804 sous le nom de République d'Haïti.

11 Dans le cadre limité de cette communication, nous nous contenterons de présenter un aperçu global des richesses architecturales subsistant en Haïti, de l'état des recherches entreprises sur le sujet et de la méthodologie qui les a guidées, pour conclure sur l'intérêt qu'elles peuvent présenter pour l'ensemble de la Caraïbe. 

cet héritage colonial de première importance saute aux yeux de l'observateur le moins averti. Constamment présent dans les campagnes, qui sont le cœur du pays, aussi bien que dans les réactions individuelles de l'habitant, il a été cependant peu étudié en Haïti même et pratiquement pas à l'extérieur.

On trouve pourtant, notamment, un peu partout dans le pays, un patrimoine architectural de premier ordre, qui, malgré son caractère exceptionnel, était encore très mal connu à l'orée des années 1980. C'est (ou plutôt, «c'était ", car les destructions se sont considérablement accentuées depuis cette époque), dans la capitale, par exemple, l'ancienne cathédrale (aujourd'hui détruite), les quelques forts qui subsistent, ou la monumentale Promenade de l'Intendance avec ses escaliers et ses fontaines..., ailleurs, dans chaque ville de province, des églises, des maisons, des ponts, des routes empierrées, des fortifications diverses, au Cap surtout, une ville tout entière qui a conservé, malgré tremblements de terre et incendies, un cachet assez semblable à celui du Vieux-Carré de la Nouvelle-Orléans avec ses maisons à galeries, ses places ornées de fontaines dont certaines portent encore la couronne royale et ses rues tracées au carré dont les noms rappellent l'Ancien Régime.

Mais c'est aussi, et surtout, disséminés dans les campagnes haïtiennes, véritable conservatoire vivant de la société d'habitation coloniale qui y prospéra dans les dernières années de l'Ancien Régime, les innombrables vestiges des constructions des quelque 8500 habitations coloniales qui tissaient au XVIII ${ }^{\mathrm{e}}$ siècle le plus important réseau rural d'exploitation des Antilles. Ces ruines des anciennes habitations coloniales, que l'habitant des campagnes a pris l'habitude de désigner sous des vocables génériques tels que vye mazi ou bagay ansien testaman ("vieilles masures» ou "choses de l'ancien temps », i.e. avant l'indépendance), constamment présentes jusque dans les coins les plus reculés du pays et dont l'accès et le repérage sont souvent difficiles, se détériorent chaque jour davantage sous les effets conjugués d'une végétation luxuriante mais aussi malheureusement de la négligence ou des déprédations humaines. Avant de subir les atteintes les plus sérieuses à une époque relativement récente, notamment au début du $\mathrm{XX}^{\mathrm{e}}$ siècle dans les grandes plaines avec l'arrivée de quelques rares usines centrales sucrières comme celle de la HASCO - Haitian \& American Sugar Company - dans la plaine du Cul-de-Sac aux portes de Port-au-Prince ou l'usine Dessalines dans la plaine des Cayes, ces vestiges de la société d'habitation coloniale étaient restés, à peu de choses près, dans l'état de destruction et d'abandon qui résultait des événements ayant entraîné la chute de la colonie dans les dernières années du XVIII ${ }^{e}$ siècle. Les récents événements liés à la période révolutionnaire ouverte avec la chute du régime duvaliériste en 1986 ont accentué un phénomène de détérioration accélérée de ces témoignages historiques qui s'étaient jusqu'ici relativement bien conservés dans des espaces ruraux quasi à l'abandon et qui, de ce fait, n'avaient donc pas subi, comme ailleurs, les effets des mutations liées à la modernisation.

\section{L'état actuel des travaux}

Deux séjours prolongés en Haïti (7 et 6 ans), nous ont permis de visiter systématiquement les sites de plus de trois cents habitations (principalement des sucreries, mais aussi des caféières et même quelques indigoteries, cotonneries ou autres guildiveries, places à vivres, chaufourneries, tuileries...), plus particulièrement dans la région de l'Ouest 
(plaines du Cul-de-Sac, de l'Arcahaye, de Léogane, de l'Artibonite, hauteurs de Pétionville, de Saint-Marc et de Montrouis...) et à un degré moindre dans le Nord (plaine et hauteurs du Cap et de Milot, île de la Tortue) et le Sud (caféières du plateau du Rochelois, plaine des Cayes, petites plaines d'Aquin, de Jacmel, des deux Goâves...). susceptibles d'étayer et de renouveler la recherche : - Recherche, recensement et exploitation systématique des ressources d'archives publiques (notamment cartographiques, mais aussi greffe, cadastre, abornements, notariat, relations diverses..., conservées au CARAN, au CAOM, dans les dépôts départementaux et locaux), et, surtout privées (les " papiers de famille ", principalement ceux d'Haïti mais aussi de la région aquitaine, mine d'informations de première main relatives à la vie et à la gestion des habitations : comptes, inventaires, correspondances de gérants... comme l'a bien montré Gabriel Debien).

18 - Exploration et reconnaissance des sites, travaux d'archéologie industrielle, enquêtes de terrain, analyse critique de la perception haïtienne actuelle (questionnements en créole). On sait le parti qu'a pu tirer, par exemple, Nathan Wachtel de ce type d'approche pour la compréhension de l'histoire coloniale espagnole à travers la "vision des vaincus $»^{6}$. L'analyse gagne indubitablement à la prise en compte de la vision haïtienne des choses, celle des vainqueurs en l'occurrence.

19 - Réalisation de monographies alliant les deux approches, première étape nécessaire avant toute tentative de synthèse.

20 Un certain nombre de publications ont rendu compte des résultats. Pour résumer rapidement, on trouvera ainsi :

21 - La présentation, dès 1981, dans deux livraisons de Conjonction, Revue franco-haïtienne de l'Institut français d'Haïti, d'une trentaine de grandes sucreries de la plaine du Cul-de-Sac, aux environs de Port-au-Prince, dont les ruines avaient été identifiées à partir de documents d'époque coloniale (cartes du XVIII ${ }^{\mathrm{e}}$ siècle, états et inventaires divers...), puis répertoriées, photographiées et resituées dans leurs contextes historique et actuel: présentation générale du quartier, situation et conditions d'accès, qualité des terres et de la production, origine du nom et informations sur les propriétaires successifs de la colonie à nos jours ainsi que sur les esclaves, événements historiques survenus sur l'habitation, état des vestiges?

22 - L'inventaire en 1986, pour l'ISPAN et l'UNESCO, publié dans une revue haïtienne, des habitations inscrites dans le périmètre du Parc Historique National qui s'étend sur trois paroisses (et partie de deux autres) voisines des sites historiques christophiens de la Citadelle Laferrière et du palais Sans-Souci. La technique d'investigation a consisté en repérages et recensement des noms de lieux (micro-toponymie) à partir de la tradition orale (questionnement en créole des habitants) confrontés ensuite aux données coloniales fournies par les cartes d'époque, l'État de l'Indemnité de Saint-Domingue (propriétés et colons) et la Description de Moreau de Saint-Méry (quartiers et cantons). Le système de conservation de la mémoire des noms, très performant, se caractérise par une grande résistance en même temps qu'une tendance à la simplification dans la transcription phonétique créole ${ }^{8}$.

23 - Les premiers résultats de l'étude récapitulative, pour les mêmes institutions, d'une vingtaine de grandes habitations qui environnent directement le Cap-Haïtien (côté plaine) sur les trois anciennes paroisses de la Petite-Anse, du Quartier-Morin et du Haut- 
du-Cap, avec le rappel des principaux sites historiques de cette région qui en est particulièrement riche (habitation Vertières et butte Charrier, les deux Bois-Caïman sur les habitations Vaudreuil et Lenormand de Mézy, les sites christophiens des habitations Duplaa, alias Les Délices de la Reine et Grand-Pont, anciennement Mac Nemara, Gallifet, point focal de l'insurrection, Bréda, lieu de naissance de Toussaint-Louverture, etc.) ${ }^{9}$.

24 - L'étude d'une partie des habitations de la région (le «transect») Madian-SalagnacAquin, dans la presqu'île du sud, essentiellement les caféières du Plateau du Rochelois, (17 sites de caféières étudiés et photographiés avec plans reconstitués ${ }^{10}$.

25 - Diverses études de terrain sur les plantations et leur environnement urbain ou rural, à l'île de la Tortue, le Cap, Fort-Dauphin, les Cayes, Saint-Louis du Sud, Jacmel, Aquin, l'Arcahaye, Montrouis, Saint-Marc, Gonaïves, Port-au-Prince ${ }^{11}$.

26 - Plusieurs monographies d'habitations de colons, principalement aquitains (sucreries Fleuriau, Clérisse, Nolivos, Laborde, Brossard-Laguehaye, caféières Navailles, Dupoy, Viaud, Châteauroux, Clérisse, indigoteries Ganderats et Pascal...), issues de l'exploitation de papiers de familles locaux dont on trouvera les références, développements ou résumés dans mes thèses et dans L'Eldorado des Aquitains ${ }^{12}$.

27 On peut tirer de l'ensemble de ces travaux quelques observations d'ordre typologique, chronologique et fonctionnel et situer le système des habitations dans ses liens avec son environnement.

\section{Typologie des habitations. Des premières exploitations : rocou, cacao, tabac et « places à vivres » primitives aux débuts de la grande exploitation : cotonneries et indigoteries}

$\mathrm{Au}$ début $\mathrm{du} \mathrm{XVII}^{\mathrm{e}}$ siècle, à l'époque où se constitue, parallèlement aux flibustiers et boucaniers, le premier noyau stable de colons - les habitants -, trois denrées d'exportation sont exploitées à Saint-Domingue à côté des vivres, manioc, patates douces, bananes, qui tiennent la plus grande place: le rocou, plante indigène dont les graines donnent une teinture rouge que les indiens Arawaks utilisaient, le cacao, introduit du Mexique à Hispaniola en 1525 par les Espagnols et que d'Ogeron tenta de développer en 1665 au Port-Margot avant sa disparition presque totale en 1717, et surtout le tabac, ou pétun dont l'usage par les Indiens avait été remarqué par Colomb dès son premier voyage en 1492. Seule cette dernière denrée connut un développement dépassant le stade artisanal de 1660 à la paix de Nimègue en 1678 surtout, assez tardivement donc à Saint-Domingue et bien après son expansion vers 1630 à Saint-Christophe, à la Barbade ou dans le Chesapeake. Culture à demi sauvage, le tabac exigeait surtout d'importantes étendues de terre à défricher et une main d'œuvre suffisamment nombreuse, parmi laquelle on comptait de nombreux engagés blancs, pour la récolte, le séchage, et la mise en rouleaux des feuilles, toutes opérations qui ne nécessitaient pas d'importantes installations techniques. Ces premières habitations, de petite taille et consistant pour l'essentiel en entrepôts de séchage ne laissèrent pas de traces dans l'architecture coloniale de SaintDomingue (celui que l'on pouvait voir sur l'habitation Gérard était une réutilisation récente de la purgerie coloniale). 
Le coton était, par ordre d'importance, la troisième denrée d'exportation à SaintDomingue et l'une des plus anciennes. Présent dès le début du XVI siècle puis quelque peu délaissé, il reprit nettement avec la Révolution industrielle pour occuper, dans la seconde moitié du XVIII ${ }^{e}$ siècle, près de $5 \%$ des terres cultivées. Le cotonnier est un arbuste qui s'élève à la hauteur de 6 à 7 pieds sur toutes les qualités de terrain. Il était planté à l'époque coloniale en quinconces. Sa qualité ne fut jamais très bonne à SaintDomingue où il subissait en outre les attaques de nombreux insectes. Toute l'industrie du coton consistait simplement à l'égrener ou à 1'épelucher comme on disait alors, c'est-àdire à séparer le duvet de la graine à l'aide d'un moulin composé de deux rouleaux cannelés actionnés par une manivelle à pied. On le tassait ensuite dans des sacs de grosse toile formant ce qu'on appelait des balles de 300 livres. Les bâtiments d'exploitation, en bois, se réduisaient à des magasins ou entrepôts qui n'ont pas laissé de traces particulières dans l'architecture coloniale malgré le grand nombre de cotonneries (ou cotonneraies), près de 800 , qui existaient encore à la fin du XVIII ${ }^{e}$ siècle, le plus souvent en association avec des indigoteries sur des terres de régions sèches.

L'indigo, enfin, herbe dont la préparation relativement complexe fournissait une teinture bleue fort en vogue à l'époque, avait été introduit à Saint-Domingue en 1676 dans la paroisse Saint-Pierre du Moustique, devenue plus tard la plaine du Nord, par les habitants français réfugiés de la baie de Samana dans l'Est de l'île. Deux variétés coexistaient dans la colonie : l'indigo "franc » et le "bâtard ». La graine qui levait en deux ou trois jours s'accommodait mieux de la sécheresse que la canne mais moins bien que le coton. Son terrain de prédilection était les petites plaines ou les vallées étroites des mornes, les sols argileux. Cette culture fut avant la canne, au début du XVIII ${ }^{e}$ siècle, la première source d'enrichissement des colons dans la mesure où elle requérait la mise en place de grands domaines, mais elle épuisait rapidement les sols et fut abandonnée dans les grandes plaines, conservant, toutefois à la veille de la Révolution près de $22 \%$ des terres en culture dans les parties sèches impropres à la canne et près de 3100 exploitations.

\section{Les grandes sucreries : l'aboutissement du système dans les plaines humides}

31 À côté des petites exploitations de l'ère pionnière, « places » à pétun, à cacao, ou à vivres, qui subsistent un peu partout, se développèrent dans la seconde moitié du XVIII ${ }^{\mathrm{e}}$ siècle les grandes sucreries qui exigeaient des regroupements de terres par dizaine de carreaux ( 1 carreau $=100$ pas de 3 pieds et $1 / 2$ en carré, soit un peu plus d' 1 ha 29 ) et d'importants ateliers atteignant souvent 2 à 300 esclaves, ainsi qu'un investissement en matériel élevé et dont la rentabilité était à beaucoup plus long terme. À la veille de la Révolution, le capital investi dans cette culture représentait $40 \%$ de toute la fortune de la colonie (en y comprenant les guildiveries, souvent adjacentes aux sucreries, où se préparait l'autre produit dérivé du sucre, le rhum), même si elle n'occupait pas plus de $14 \%$ de la surface cultivée. Les principaux sites de culture de la canne étaient les plaines du Nord, surtout autour du Cap, les plus anciennement établies, qui fournissaient $75 \%$ du sucre terré, le Cul-de-Sac, près de Port-au-Prince, et ses satellites de l'Arcahaye et de Léogane, qui donnaient les 3/4 du sucre brut, et enfin la plaine des Cayes dans le Sud. L'Artibonite et quelques autres plaines de moindre importance (Jean-Rabel, Jacmel, Jérémie, Saint-Marc, Nippes, Aquin, Cavaillon, Saint-Louis, le Plateau Central...) complétaient la production. 

nombreuses innovations techniques, de l'époque du père Labat à celle de Belin de Villeneuve, et des plus belles installations en bâtiments d'habitation et surtout d'exploitation qui, avec quelques variantes régionales, suivent à peu près toutes le même modèle général tel qu'on peut le reconnaître sur les plans, gravures et inventaires d'époque ainsi que sur les vestiges actuels.

La partie habitée avait pour centre la grand-case, maison du colon, si possible en position dominante (pour mieux surveiller) et au vent des bâtiments d'exploitation (pour éviter tous risques et désagréments tels que bruits, odeurs, incendies). Elle était le plus souvent en bois, sauf dans le Nord, sur socle de maçonne, les murs bousillés entre poteaux, sans étage, entourée d'une galerie, le toit en essentes (planchettes teintes en noir imitant l'ardoise), tuiles ou ardoises pour les plus riches, le sol carrelé ; parfois sur une cave voûtée. La cuisine, sommaire, était à l'écart pour éviter les risques d'incendies. Le dispensaire, pompeusement baptisé hôpital, suivait un modèle traditionnel en trois parties : chambres pour hommes et femmes séparées par une salle de consultations et équipées d'une barre et d'organeaux pour immobiliser les malades. Ce bâtiment était le plus souvent en bois, poteaux en terre, murs clissés et bousillés, carrelé au sol et couvert en pailles, essentes ou tuiles. Des cases séparées servaient à l'isolement des malades contagieux. À proximité se trouvaient généralement les logements de l'hospitalière, des domestiques, du cuisinier et souvent une case pour le logement des hôtes ; un poulailler, un colombier, des magasins et entrepôts, les logements des économes et guildiviers blancs, le clocher pour appeler les esclaves au travail, parfois un cachot voûté en maçonne, un four à chaux, des bâtiments abritant machoquèterie (forge), tonnellerie et charronnerie, des parcs à bêtes, puits, abreuvoirs... Une grande allée bordée d'arbres menait à un portail aux piliers monumentaux (surtout dans le Nord) fermé par une grille en fer forgé. Le colon mettait sa fierté dans la beauté de cette entrée.

sition des bâtiments d'exploitation, situés sous le vent, suivait les étapes de la fabrication du sucre. Tout d'abord, lorsqu'il y avait suffisamment d'eau, un long aqueduc d'une centaine de mètres ou plus, aux arches monumentales, alimenté par des canaux... qui conduisait l'eau, dans sa dalle encadrée par les mâchoires, jusqu'au moulin où elle tombait sur la grande roue avant de s'évacuer par la taillevanne au fond de la fosse. Un escalier permettait d'accéder au sommet de la vanne d'admission d'eau. La case à moulin, en dur, abritait les trois rôles (rouleaux en bois cerclé dur cerclés de fer), à travers lesquels on pressait la canne, et s'accompagnait d'un parc à cannes. De la case à moulin, le vesou ou vin de canne s'écoulait par un canal vers les deux équipages de cinq à six chaudières de la sucrerie proprement dite (ou chaufferie), reconnaissable à ses cheminées et à ses fourneaux alimentés par la combustion de la bagasse (résidu de canne pressée) et souvent doublée d'une raffinerie. Un peu plus loin, les purgeries où le sucre se purifiait dans des formes, affectaient traditionnellement la forme d'un $\mathrm{U}$ ou d'un $\mathrm{L}$ et étaient le plus souvent en bois, ce qui explique la rareté de conservation de leurs ruines. On portait enfin le sucre à l'étuve, souvent double, sorte de haute tour hermétique, parfois sommée d'un clocheton, où les pains de sucre étaient séchés pendant une quinzaine de jours sur des planchers en étages par un feu continu entretenu dans un four parallélépipédique en fonte.

Sur les habitations dépourvues d'eau (ou pour compléter le moulin hydraulique), on trouvait encore un moulin à bêtes, souvent double, avec son chemin de ronde surélevé sur une base en maçonne percée de belles entrées voûtées pour y apporter la canne, son toit

In Situ, 20 | 2013 
en forme de chapiteau conique, sa rampe d'accès pour les bêtes, son parc à cannes semicirculaire, et ses rôles sur socle en maçonne, horizontaux (ou «à l'anglaise») ou verticaux. La guildiverie où se fabriquait le tafia était en général un bâtiment en dur adossé à l'aqueduc. La plupart des guildives que l'on voit actuellement en Haïti et ailleurs se sont installées sur les ruines des bâtiments coloniaux.

Le quartier des esclaves, à bonne distance sous le vent, était constitué d'un alignement symétrique de cases à nègres en torchis, plus rarement en bois et même parfois importées «en préfabriqué » d'Amérique du Nord, dont il ne reste pratiquement plus d'exemples d'époque (un rare exemple en était encore visible dans les années 1980 sur l'habitation Péra). En revanche, on trouve encore aujourd'hui en grande quantité des vestiges de sucreries, aqueducs, moulins, étuves, dans les grandes plaines à sucre d'Haïti. Certains sont imposants, surtout dans le Nord et dans l'Ouest, mais il est rare qu'une habitation complète ait été conservée, comme à Pivert, Delugé ou Guillon, les trois dernières en activité dans les années 1980.

\section{Les caféières (ou caféteries) : le nouveau front pionnier des mornes}

Dernière exploitation importante chronologiquement, mais la seconde en production et en rendement financier, la culture du café à Saint-Domingue n'apparut que tardivement, dans les dernières années de la colonie, le dernier quart du XVIII ${ }^{e}$ siècle surtout, bien après les petites Antilles. Elle constituait alors le front pionnier, après les règnes successifs du tabac, de l'indigo et de la canne à sucre.

Les premiers plants de café avaient été apportés par les Jésuites en 1725, en provenance de la Martinique, sur leur habitation du Terrier-Rouge dans le Nord. L'année suivante, M. de Nolivos les introduisit dans l'Ouest sur son habitation de Léogane mais il fallut attendre 1738 pour voir se monter les premières caféières dans la paroisse du Dondon et la véritable expansion de cette culture ne commença qu'en 1770. La production qui n'était que de 7 millions de livres en 1755 atteignait 77 millions en 1789 (un peu plus que le sucre), occupant $60 \%$ des terres cultivées.

Le caféier, arbuste de 6 à 7 pieds de hauteur que l'on plantait en quinconces sur un terrain préalablement défriché, un bois neuf, à raison de 3 à 4000 pieds au carreau, préférait les terres des mornes, entre 300 et 900 mètres d'altitude. L'exploitation nécessitait des bâtiments moins considérables que ceux des sucreries et les colons caféiers étaient d'ailleurs en général moins fortunés et considérés, mais la présence de pierres dans les mornes, la nécessité de se protéger du froid et les multiples opérations de lavage, broyage et séchage nécessaires à la préparation du café entraînèrent la construction de nombreuses installations en dur sur les caféières.

Le groupe des bâtiments d'habitation comprenait habituellement une grand-case située en position dominante, au sommet du morne; non loin mais séparée pour éviter les risques d'incendie, une cuisine avec la cheminée et son four à pain qui généralement ont subsisté ; un quartier des esclaves constitué d'un ou deux bâtiments en dur divisés en petites cases familiales et un hôpital en trois parties. Pour la préparation du café on utilisait d'abord un moulin à grager, c'est-à-dire à déceriser, qui était en bois et actionné à la main par une équipe d'une douzaine d'esclaves. On lavait ensuite le café dans un bassin circulaire où les impuretés étaient entrainées par une eau courante et on l'égouttait sur 
une plate-forme où il était étendu et exposé pendant 24 heures. Sur la plupart des caféières un grand bâtiment d'exploitation en trois parties, souvent bien conservé et parfaitement reconnaissable par ses bassins et ses conduites d'eau, servait à ses trois opérations. La phase suivante consistait en séchage des grains en parchemin sur des glacis maçonnés au centre desquels se trouvaient de petits bassins circulaires, les bassicots, vers lesquels on poussait le café le soir pour le protéger de l'humidité en le recouvrant. Les grains passaient ensuite au moulin à piler dont la roue verticale, actionnée par des mules, détachait les parchemins sans écraser les fèves en tournant dans une gorge circulaire en pierre de 10 à 12 pieds de diamètre. Ces moulins étaient parfois abrités par une case. On utilisait enfin un moulin à vanner, actionné à la main, dont la soufflerie faisait s'envoler les parchemins, avant de trier les grains sur de longues tables. Ces dernières installations, en bois, n'ont pas subsisté. Des magasins et entrepôts, parfois bien conservés, venaient compléter l'ensemble de ces bâtiments d'exploitation.

41 C'est dans les quartiers montagneux que l'on trouve le plus de vestiges des habitations caféières, principalement dans les massifs du Nord (Dondon, Marmelade, GrandeRivière...), dans les chaînes des Matheux (au Fond-Baptiste notamment) et des Cahos au centre du pays, et dans le Sud, sur le Plateau du Rochelois et les hauteurs du massif de la Hotte.

\section{L'environnement de l'habitation : architecture civile, réseau routier et ponts, distribution des eaux, barrages et fontaines}

42 L'exploitation des habitations nécessitait, par ailleurs, toutes sortes d'infrastructures dont beaucoup de traces sont encore visibles aujourd'hui. Les routes de la colonie avaient été construites par portions, sans plan général, selon les besoins du moment. Elles se divisaient en chemins royaux, paroissiaux et privés, ces derniers faisant communiquer les habitations avec les embarcadères par le système de la corvée fournie par les habitations selon leur importance. Les routes principales existaient dès le début du XVIII ${ }^{e}$ siècle. On doit au gouverneur Larnage l'ouverture de chemins dans les passes difficiles, principalement dans le Sud. La liaison Port-au-Prince-le Cap connut en 1751 une sensible amélioration entreprise par Vaudreuil mais le passage du col de Puilboreau se faisait par une section impraticable aux voitures et difficile même à cheval, dénommée Les escaliers, sur plus de deux kilomètres. À partir de 1788 un détour permit d'améliorer la communication en la rendant carrossable et un service de voitures fut établi. Un règlement de 1781, qui ne put être observé régulièrement, avait fixé la largeur des chemins à 10 pieds dans les mornes. On trouve encore aujourd'hui de nombreuses traces de ce réseau routier sous forme de tronçons empierrés ou pavés qui ont résisté à la destruction jusque dans les endroits les plus reculés. Les routes actuelles reprennent le plus souvent le tracé de l'époque.

Le passage des rivières et ravines se fit longtemps à gué ou, dans le cas de cours d'eau importants comme l'Artibonite, par bac (à la Petite-Rivière). Le premier pont de pierre construit dans la colonie le fut à Saint-Marc en 1785. Il avait 28 pieds d'ouverture sur 33 de large et fut suivi, en 1786, par le pont Larnage (devenu plus tard Pont-Rouge) à l'entrée de Port-au-Prince (invisible aujourd'hui sous l'urbanisation). Le grand pont-canal sur l'Artibonite (gravure existante), projeté pour remplacer le bac en 1790, n'eut pas le temps 
d'être réalisé et le pont de Bréda au Haut-du-Cap, en bois et remplacé par un pont encore en état aujourd'hui portant une date post-coloniale, resta le plus grand pont de la colonie. Dans le Cul-de-Sac, le pont colonial de Charrier sur le coursier Est de la Rivière Grise est bien conservé. Une ordonnance de 1788 enjoignit aux propriétaires de faire construire des ponts en maçonnerie de 30 pieds de large sur les fossés d'écoulement des eaux de leurs habitations. C'est ainsi qu'on trouve aujourd'hui, dans les plaines, quantité de petits ponts de belle facture qui se sont bien conservés.

C'est encore aux administrateurs Larnage et Maillard que l'on doit la mise à l'étude d'un remarquable réseau de distribution des eaux qui changea le sort de nombreuses terres réputées stériles. Des canaux, barrages et bassins de distribution furent établis progressivement, principalement dans les grandes plaines à sucre des Cayes, du Cap et du Cul-de-Sac. Dans l'Artibonite, après les études de Trembley, un autre habitant, Bertrand de Saint-Ouen, projeta et commença à exécuter un plan révolutionnaire d'arrosement par un siphon et une pompe à vapeur qui entra en fonctionnement le 11 novembre 1786 en présence du Gouverneur et dont les ruines, étampées Perrier frères, Chaumont, existaient encore deux siècles plus tard. Elle prenait l'eau à 12 pieds au-dessous des plus basses eaux et pouvait l'élever à 21 pieds, arrosant 2000 carreaux. Mais cette innovation resta sans lendemain par suite de la résistance des habitants voisins. L'une des plus remarquables de ces réalisations fut le Bassin Général de la Grande Rivière du Cul-de-Sac, commencé en 1773 et achevé en 1784, qui fonctionne encore. Il permettait d'arroser 7988 carreaux dépendant de 58 sucreries. Un peu plus loin, le Bassin Joly, sur la Rivière Blanche, de dimensions plus modestes desservait 4959 carreaux. Dans la plaine des Cayes, après les premiers travaux de l'Intendant Maillard à Torbeck, le barrage le plus remarquable fut celui réalisé par le bigourdan d'Avezac de Castéra qui existe encore sous le nom de canal Davezac. Dans le Nord, autour du Cap, où l'abondance des pluies et des cours d'eau ne nécessitait pas la mise en place d'une distribution générale, les habitants se contentèrent d'établir des batardeaux dans le lit des rivières. À l'Arcahaye et à Léogane on procéda par saignées et dérivations, sans grands travaux d'art. Après diverses expériences, l'ingénieur Ricord avait conclu en 1739 à l'impossibilité d'arroser la plaine d'Aquin et les essais tentés dans la Savane Désolée de la plaine des Gonaïves furent aussi abandonnés, mais dans beaucoup d'autres endroits les eaux des rivières qui se perdaient sans profit furent captées, canalisées, retenues et distribuées aux habitations sous l'autorité de syndics des eaux.

Pour l'alimentation en eau potable des villes on capta des sources comme celles de Turgeau, Martissant et la Charbonnière dans les hauteurs de Port-au-Prince. Cela permit l'aménagement de fontaines sur les places publiques des villes dont la plupart ont disparu ou ne fonctionnent plus. On en trouve quelques vestiges au Cap (fontaine de la place Toussaint-Louverture, ex Place Royale) et dans ses alentours (très belle fontaine Ducommun avec sa vasque et ses armes) ainsi que sur l'ancienne Promenade de l'Intendance à Port-au-Prince.

\section{Architecture urbaine, militaire et religieuse}

Toute une architecture urbaine, militaire et religieuse s'était également développée avec l'essor de la grande plantation et à sa suite. 
47 Dans les villes, le tracé des rues, au carré, est toujours colonial, particulièrement remarquable à Fort-Liberté (ci-devant Fort-Dauphin) où l'urbanisme colonial avait prévu très large pour cette place forte appelée à se développer. Mais c'est le Cap-Haïtien, anciennement Cap-Français, qui présente les plus grandes richesses. Ravagée par les incendies historiques de 1793 (affaire Galbaud-Sonthonax, affrontement entre le gouverneur, soutenu par les colons, et le commissaire de la République qui fait appel aux noirs) et 1802 (sur ordre de Christophe à l'arrivée de l'expédition de reconquête bonapartiste) et enfin par le tremblement de terre de 1843, la ville a cependant conservé intacte sa base coloniale : rues au carré dont certaines ont conservé leurs plaques, îlets regroupant quatre maisons d'angle autour d'une cour commune et à l'intérieur desquelles on retrouve en rez-de-chaussée puits, fours à pain, escaliers, carrelages et distribution des pièces d'époque. Certains monuments et édifices publics ont également résisté : portail de la Providence du Cap (l'hôpital), casernes coloniales (aujourd'hui hôpital SaintJustinien), ponts, traces de remparts, boulangerie du roi... etc. Les cheminées, solidement construites, que l'on découvre en position dominante, de l'école des Pères, sont aussi particulièrement remarquables. Un inventaire et schéma directeur du Cap pour la préservation du centre historique étaient en cours sous la direction de l'ISPAN (Institut de Sauvegarde du Patrimoine National) en 1986.

Les nombreuses fortifications coloniales subsistantes mériteraient aussi de longs développements. La plupart sont côtières, rappelant la deuxième phase de l'évolution du système défensif local, après la défense maritime par escadres et avant la stratégie de repli sur des places fortes de l'intérieur, plus spécifiquement haïtienne mais initiée par le gouverneur basque Armand de Belzunce. Le Nord est particulièrement riche dans ce domaine avec les fortifications de Fort-Liberté (notamment le fort La Bouque et le Fort Dauphin dont la poudrière est bien conservée), celles du Cap surtout (forts Saint-Joseph, aux Dames, et surtout Picolet, en bon état de conservation), de Port-de-Paix (Grand-Fort et Petit-Fort de la Pointe des Pères) et du Môle Saint-Nicolas, le Gibraltar de la Caraibe (casernes, poudrière monumentale, batteries diverses). À Port-au-Prince, où la batterie Sainte-Claire a été récemment détruite, les forts ou batteries du Bel-Air, À Rebours et SaintJoseph ont disparu sous les aménagements successifs, mais les ruines du Fort-l'Islet construit sur un îlot de la rade et les forts Bizoton et Mercredi sont encore nettement visibles. Un autre point intéressant pour l'étude des fortifications coloniales est la baie de Saint-Louis, dans le Sud, fortifiée par la Compagnie, où l'on peut voir les ruines du GrandFort-Saint-Louis sur une île de la rade, et de la Batterie de la Compagnie sur la pointe des Oliviers, assez bien conservée. Quant à l'île de la Tortue elle ne conserve que quelques pans de murs de la Batterie Haute réaménagée par Labatut en 1776, des canons abandonnés sur les sites des deux batteries de la rade et de la Tour d'Ogeron, et quelques lignes de pierre des bastions établis autour du célèbre rocher connu successivement sous le nom de Refuge et de Fort Levasseur à l'époque flibustière.

Quant à l'architecture religieuse, elle avait pour plus beau fleuron il y a peu encore l'ancienne cathédrale du Port-au-Prince construite après le tremblement de terre de 1770 et dont la restauration était toujours en cours dans les années 90 lorsqu'elle fut entièrement détruite par un incendie criminel lié aux soubresauts politiques du pays. On y trouvait notamment un bel autel en marbre offert par Louis XVI, la plaque tombale du Grand Juge Dom Sabourin, d'imposants piliers en acajou massif et de beaux travaux de maîtres charpentiers et menuisiers à l'intérieur, notamment un escalier et une chaire. Cet édifice était le seul vestige colonial de la ville avec la Promenade de l'Intendance voisine, 
ses deux fontaines et ses escaliers, depuis la disparition, dans le même quartier au début du siècle, de l'abreuvoir (Bassin Cheval), et, plus récemment, de la dernière aile subsistante de l'Hôtel de la Marine, abattue en 1985. De nombreuses églises de province sont également remarquables, à Torbeck, à la Petite-Anse, au Cap et environs. De la cathédrale de SaintMarc il ne reste qu'une tour ruinée dont l'architecture hexagonale rappelle la tradition des bâtisseurs gascons, et de celle du Cap - dont la façade rappelle celle de la cathédrale de Montauban selon Armand Viré - seulement les bases sur lesquelles a été reconstruit l'édifice actuel. On n'aurait garde d'oublier enfin les nombreux cimetières des villes ou campagnes qui demanderaient un inventaire détaillé et réservent parfois des surprises comme celui de Jacmel où l'on trouve deux tombes aux belles plaques de marbre portant l'inscription natif (native) d'Afrique ainsi que celle d'Horace-Camille Desmoulins, fils du célèbre révolutionnaire français. À Port-au-Prince, l'ancien Cimetière Intérieur (aujourd'hui École Sainte-Anne) abrite le très beau mausolée du gouverneur-comte Thérèse Charpentier d'Ennery à deux pas de la tombe du héros de l'indépendance nationale, JeanJacques Dessalines. C'est également là que fut inhumé (sous l'actuelle église Sainte-Anne) le tristement célèbre conventionnel Billaud-Varenne qui avait fini ses jours en Haïti après son exil guyanais.

\section{Conclusion}

50 Au-delà de leur valeur purement esthétique et de la charge émotionnelle qui les habite, ces vestiges représentent pour Haïti un patrimoine architectural exceptionnel et irremplaçable que bon nombre de pays neufs pourraient lui envier. Véritables lieux de mémoire de l'esclavage dont ils ont constitué le cadre de la vie quotidienne pour la grande majorité de ses victimes et acteurs, ils sont en même temps les témoins de la longue histoire commune franco-haïtienne et l'expression d'un type de société préindustrielle pionnière, à dominante agro-commerciale - la société créole esclavagiste d'économie de plantation fondée sur l'exploitation extrême d'hommes privés de leur liberté - dont Saint-Domingue constituait la pointe la plus avancée et qui a façonné toute cette région de la Caraïbe non seulement dans ses paysages et ses réalisations mais aussi bien plus profondément peut-être dans les valeurs humaines, les mœurs et les coutumes hérités d'une histoire de labeur et de souffrance. Après les troubles qui marquèrent la fin de la colonie et l'indépendance d'Haïti, la diaspora des anciens colons de Saint-Domingue et de leurs esclaves dans la Caraïbe a, en effet, contribué à répandre ce modèle dans les pays avoisinants - et plus particulièrement à Cuba (pour le sucre et le café), à la Jamaïque (pour le café) et à la Nouvelle-Orléans (pour le coton) -, justifiant ainsi aujourd'hui la nécessité d'un caractère transnational à donner aux recherches à venir. Au-delà de l'évidence de la médiation dominguoise dans la diffusion du modèle français, puis antillais en général, voire "américain", l'élargissement de la mise en perspective comparative dans un premier temps à d'autres pays de la Caraïbe (Jamaïque, Cuba, ÉtatsUnis, République Dominicaine...) devrait encore permettre d'utiles avancées de la connaissance.

51 Un premier travail de grande utilité pour les échanges entre chercheurs pourrait être la constitution d'un glossaire quadrilingue (français, espagnol, créole, anglais) des termes usuels du lexique de l'habitation, puis d'un répertoire des principales sources et de leur localisation. Il conviendrait ensuite, dans un second temps de croiser les travaux effectués dans différentes îles pour en tirer des synthèses à l'échelle caribéenne, avant 
d'affiner les résultats dans le sens d'une distinction entre les divers apports européens (et leurs lointaines origines) et ceux issus de l'incorporation d'éléments plus spécifiquement locaux relevant d'une certaine créolisation.

\section{BIBLIOGRAPHIE}

BUTEL, Paul. «L'essor de l'économie de plantation à Saint-Domingue dans la seconde moitié du XVIII ${ }^{e}$ siècle ». Bulletin du Centre d'Histoire des Espaces Atlantiques, n 7,1995, p. 61-76.

CAUNA, Jacques de :

- Au temps des Isles à Sucre. Histoire d'une plantation de Saint-Domingue au XVIII siècle. Paris : Karthala, 1987, 288 p., condensé de la thèse de doctorat de III ${ }^{\mathrm{e}}$ cycle intitulée Une habitation de Saint-Domingue à la fin du XVIII e siècle : la sucrerie Fleuriau de Bellevue, Université de Poitiers, 1983, 765 p. en 2 vol., dir. Jean Tarrade.

- « Vestiges de sucreries dans la Plaine du Cul-de-Sac ». Conjonction, 1981, n¹49, p. 63-104, et $1985, n^{\circ} 165$, p. 4-32.

- « Mémoire des lieux, lieux de mémoire : quelques aperçus sur la toponymie haïtienne et ses racines historiques, $1986 »$. Chemins Critiques, Port-au-Prince, 1990, n4, p. 125-140.

- Paysans, systèmes et crises. Travaux sur l'agraire haïtien. Pointe-à-Pitre et Port-au-Prince : SACAD (Université des Antilles et de la Guyane) et FAMV (Université d'État d'Haïti), 1993, collaboration tome 1, Histoire agraire et développement, $365 \mathrm{p}$.

- Haït, route 2004, Écomusée du Nord, rapport PNUD/UNESCO, projet 95/010, juin 1996, p. 1-49, annexes p. 50-69.

- L'Eldorado des Aquitains. Gascons, Basques et Béarnais aux Iles d'Amérique (XVII ${ }^{e}$-XVIII ${ }^{e}$ siècles). Biarritz : Atlantica, 1998, 541 p.

- La colonisation française aux Antilles. Les Aquitains à Saint-Domingue (XVII ${ }^{e}$-XVIII ${ }^{e}$ siècles). Thèse pour le Doctorat d'État, Université Paris IV-Sorbonne, direction J.P. Poussou, 2000, 3 vol. (429 p. + 419 p. + travaux).

CRATON, M. and WALVIN, J. A jamaïcan plantation : the history of Worthy Park, 1670-1970. Cambridge University Press, 1978.

DEBIEN, Gabriel. Notes d'histoire coloniale (plus de 200 titres dont de nombreuses monographies d'habitations).

FREYRE, Gilberto. Maîtres et esclaves (traduction française). Paris : Gallimard, 1952.

HIGMAN, Barry. Jamaica surveyed. Plantation maps and plans of the eighteenth and nineteenth centuries. Kingston, 1988.

MORENO FRAGINALS, Manuel. El ingenio : el complejo economico-social cubano del azucar, 1760-1860. La Havane, 1976.

PLUCHON, Pierre. «L'économie d'habitation à Saint-Domingue ». Revue d'Histoire Maritime, 1, 1995, p. 197-241. 
WACHTEL, Nathan. La Vison des vaincus. Les Indiens du Pérou devant la conquête espagnole. Paris : Gallimard, 1971.

Pour la bibliographie de l'auteur, voir en complément :

http://www.esclavages.cnrs.fr (site du Centre International de Recherche sur les Esclavages)

http://jdecauna.over-blog.com (blog de la Chaire d'Haïti à Bordeaux)

Références : Fonds Jacques de Cauna. Mémoire et patrimoine de l'esclavage en Haïti

Conception et rédaction : Jacques de Cauna, Myriam Cottias

Coordination et recherches iconographiques : Jacques de Cauna, Myriam Cottias, Véronique

Ikabanga

Conception et réalisation graphique : Frédéric Eckly, Véronique Ikabanga

CIRESC/CNRS Images

\section{NOTES}

1. - Ce texte reprend en grande partie la communication de Jacques de Cauna, «Aperçus sur le système des habitations aux Antilles françaises. Vestiges architecturaux et empreinte aquitaine en Haït (ancienne Saint-Domingue) », publiée dans Le monde caraïbe. Échanges transatlantiques et horizons post-coloniaux (dir. Christian Lerat), MSHA Bordeaux, 2001, p. 133-152. On en trouvera une version en langue anglaise dans une autre communication présentée en juin 2004 à la Brown University, Providence, Rhodes Island (USA) sous le titre "Vestiges of the Built Landscape of Pre-revolutionnary Saint-Domingue ", p. 21-48, iconographie (16), et publiée dans The world of the Haittian Revolution, edited by David Geggus and Norman Fiering (John Carter Brown Library), coll. Blacks in diaspora, Indiana University Press, Bloomington (USA), 2009.

2. - À côté des indispensables travaux de Gabriel Debien pour la Caraïbe francophone (ses Notes d'histoire coloniale, plus de 200 titres dont de nombreuses monographies d'habitations et sa synthèse, Les esclaves aux Antilles françaises. Basse-Terre, Fort-de-France : Sociétés d'Histoire de la Guadeloupe et de la Martinique, 1974), voir en particulier les remarquables études de : FREYRE, Gilberto (Brésil). Maîtres et esclaves (traduction française). Paris: Gallimard, 1952. MORENO FRAGINALS, Manuel (Cuba). El ingenio : el complejo economico-social cubano del azucar, 1760-1860. La Havane, 1976. HIGMAN, Barry (Jamaïque). Jamaïca surveyed. Plantation maps and plans of the eighteenth and nineteenth centuries. Kingston, 1988. CRATON, M., WALVIN, J. A jamaïcan plantation : the history of Worthy Park, 1670-1970. Cambridge University Press, 1978.

3. - On les nommait ainsi par dérision en référence à leur contrat d'engagement qui était de trois ans.

4. - Sur ce point, voir : CAUNA, Jacques de. Au temps des Isles à Sucre. Histoire d'une plantation de Saint-Domingue au XVIII ${ }^{e}$ siècle. Paris : Karthala, 1987, condensé de la thèse de doctorat de $3^{\mathrm{e}}$ cycle intitulée Une habitation de Saint-Domingue à la fin du XVIII siècle: la sucrerie Fleuriau de Bellevue. Université de Poitiers, 1983, 2 vol., dir. Jean Tarrade; BUTEL, Paul. "L'essor de l'économie de plantation à Saint-Domingue dans la seconde moitié du XVIII ${ }^{\mathrm{e}}$ siècle ». Bulletin du Centre d'Histoire des Espaces Atlantiques, n 7, 1995, p. 61-76; PLUCHON, Pierre. «L'économie d'habitation à SaintDomingue ». Revue d'Histoire Maritime, n 1, 1995, p. 197-241.

5. - CAUNA, Jacques de. L'Eldorado des Aquitains. Gascons, Basques et Béarnais aux Iles d'Amérique (XVII ${ }^{e}$-XVIII ${ }^{e}$ siècles). Biarritz: Atlantica, 1998. C'est ce parti qui a été pris dix ans plus tard dans la conception et l'élaboration de l'exposition permanente du Musée d'Aquitaine, et notamment pour la salle éponyme consacrée à la société d'habitation dans laquelle est présentée, entre autres, une maquette de la sucrerie Nolivos. Voir le catalogue bilingue: HUBERT, François, 
BLOCK, Christian, CAUNA, Jacques de. Bordeaux au XVIII e siècle, le commerce atlantique et l'esclavage. Bordeaux : Éd. Le Festin, 2010.

6. - WACHTEL, Nathan. La Vision des vaincus. Les Indiens du Pérou devant la conquête espagnole. Paris : Gallimard, 1971.

7. - « Vestiges de sucreries dans la Plaine du Cul-de-Sac ». Conjonction, 1981, n 149, p. 63-104, et 1985, n 165, p. 4-32. Les principales habitations étudiées ont été regroupées par anciens quartiers ou cantons: Bellevue (Caradeux, Fleuriau, Châteaublond, Cazeaux, Goureaud, Dumornay, Mocquet, Bauduy ML, Soissons, Greffin, Bauduy, Pernier, Frère), Petit-Bois (Duval, Borgella, Daulnay, Courpon, Jumécourt, Noailles, La Ferronays, Dargout, Blanchard, Robert, Michaud, Lucas), La Grande-Raque (Dumée ou Caradeux La Caye, Digneron, Rocheblanche, Drouillard, Turbé), Les Varreux (Santo, Damiens, Lilavois, Bonrepos, Beudet, Le Meilleur, Le Roux ou Nolivos, Despinose), La Grande-Plaine (Jouon ou Jonc, Vaudreuil Grande-Plaine, La Tremblaye, Pera ou Peyrac, O'Gorman, Baugé ML, La Mardelle, Jacquet, Boën, Chambon, Bassin Joly).

8. - CAUNA, Jacques de. "Mémoire des lieux, lieux de mémoire: quelques aperçus sur la toponymie haïtienne et ses racines historiques », 1986. Chemins Critiques, Port-au-Prince, 1990, n • 4, p. 125-140. Enquête de terrain à ambition modélisante menée en compagnie d'une géographe française et de trois chercheurs haïtiens (archéologue, ethnologue et cartographe) de l'ISPAN (Institut de Sauvegarde du Patrimoine National) dans le cadre d'un projet PNUD/UNESCO (consultant national, 1986), présentée à la table ronde L'histoire des sociétés coloniales antillaises, 1990, Paris : École des Hautes Études en Sciences Sociales. Habitations et sites visités : Milot, Sans-Souci, citadelle Laferrière, les Ramiers, caféières Ginet, Hériveaux, Junca, Sèze, sucrerie Grand-Pré...

9. - Dans le cadre du rapport PNUD/UNESCO pour la création d'un écomusée du Nord en Haïti (projet 95/010 Haïti, Route 2004, consultance externe, juin 1996, p. 1-49, et annexes p. 50-69. Sources : archives, plans et cartes d'époque, terrain, papiers de famille... Principales habitations et sites étudiés: La Voûte, Saint-Martin, Pères de la Charité, Vertières, Charrier, Bréda, Ducommun, Le Normand de Mézy, Bois-Caïman, Dhéricourt, Vaudreuil, Lombard (Haut-du-Cap, Plaine-du-Nord, Morne-Rouge), Desglaireaux, Duplàa ou Galman Dupla ou Les Délices de la Reine, Charritte, Détrel ou Destreilles, Chastenoye, Nazon, bourg du Morin, Guillodeu, Clérisse, Héritiers Le Fèvre, Grand-Pré ou La Victoire, Magdeline ou Portelance, Beaujau ou Bourjo, Lalande-Gayon (Quartier-Morin), Galliffet (les trois sucreries : Grande Place, La Gossette et Desplantes), Bongars, le bourg ou embarcadère, Saint-Michel, Pécome, Choiseul, Mazères, Baubert, Le Fêvre, Montégut, Gabriac Saint-Paulet, Brossard, Beaunay de Boishimont et Beaunay Dutôt (La Petite-Anse).

10. - Dans le cadre du projet Madian-Salagnac (expert local, Mission de coopération française), résultats publiés dans PILLOT, Didier et CAUNA, Jacques de. "Histoire agraire et développement ", tome 1. Paysans, systèmes et crises. Travaux sur l'agraire haïtien. Pointe-à-Pitre et Port-au-Prince : SACAD (Université des Antilles et de la Guyane) et FAMV (Université d'État d'Haïti), 1993. Habitations étudiées: Barbier, Brun, Cavillier, Colombel, De Renoncourt, Febvé, Fort, La Barre, Lafargue, Lavau, Leblanc, Leige, Lorquet, Masson, Mussotte, Pottier, Godets ou Regnauld, Rey, De Ronseray, Sainte-Croix, Salaignac, Séguineau, Sentou, Spinefort, Tauzin, Viaud, Villedrouin, Violette, Bérot, Berquin, Bourricaud, Brondeau, Coututier, Chantrel, Desraignes ou Javel, Destrez, Lafargue, Lafosse, Monneron, O’Rourke, Pagès, Roux, Saint-Mégrin, Sainton, Teinturier, Turlin, Vivent, Chasseau ou Chassereau, Catin, Bannac, Perrien ou Perigny, Jondeux, Flipo ou Phellipeaux, Jacquot, Bidouze.

11. - Dans le cadre des activités du Centre de Recherches Historiques de l'Institut Français d'Haïti (direction), quelques habitations et sites visités et étudiés : Basse-Terre, Cayonne, Palmistes, La Croix, la Tour d'Ogeron, le Rocher ou Fort-Refuge, ou Fort Le Vasseur, la Batterie d'En Haut ou Batterie Labatut, l'hôpital, les batteries de la rade (île de la Tortue), Fort Picolet, batterie Magny, Fort-aux-Dames, Platon François, Lafargue, Fort Poirier, Fort Bély, Cormier, Petit Port Français, Pointe-à-Honorat, Dupaty (Bande du Nord et l'Acul), les Gonaïves, la Grande-Saline, la Savane 
Désolée, Labadie-Lacroix, la Crête-à-Pierrot, le Palais des 365 Portes, Bretton Deschapelles (Artibonite), Prince, Garesché, Deschapelles, Torcelles, Fauveau, Bailleul, Manègue, Cabaret, Cazal (Le Boucassin), Saintard, Roberjot, Vergès, Poy La Ravine, Poy La Générale, Imbert, Vignier, BoisNeuf, Guibert (Les Vases, l'Arcahaye, les Matheux), Dupin, Vendôme, Cadenet, Ogier, de Lugé, Lanzac, Pierre Payen (les «sept moulins » de Montrouis), Pivert, Guillon ou Nonès, La Roussette, Moreau Grande-Place (hauts de Saint-Marc), Gérard, Pémerles, Picot (Plaine du Fond de l'île-àVache ou des Cayes), Glaize, Darbonne, Mendès-France, l'Acul, le Fort Nolivos (Léogane et les Goâves)..., etc. La plupart des données recueillies sur ces sites sont restées inédites, à l'exception du rapport de mission scientifique publié dans : CAUNA, Jacques de (dir.). Conjonction, $\mathrm{n}^{\circ}$ 174-175 ( spécial Île de la Tortue), $3^{\mathrm{e}}$ et $4^{\mathrm{e}}$ trim, 1987.

12. - Outre L'Eldorado des Aquitains... (CAUNA, Jacques de. L'Eldorado des Aquitains. Gascons, Basques et Béarnais aux Iles d'Amérique (XVII ${ }^{e}$-XVIII ${ }^{e}$ siècles). Biarritz: Atlantica, 1998), qui reprend La colonisation française aux Antilles. Les Aquitains à Saint-Domingue (XVII ${ }^{e}$-XVIII ${ }^{e}$ siècles), thèse pour le Doctorat d'État, Université Paris IV-Sorbonne, direction J.P. Poussou, 2000, 3 vol., voir aussi : CAUNA, Jacques de. Au temps des Isles à Sucre. Histoire d'une plantation de Saint-Domingue au XVIIIe siècle. Paris : Karthala, 1987.

\section{RÉSUMÉS}

Haïti, première république noire du monde est aujourd'hui encore un véritable conservatoire du patrimoine historique de l'économie esclavagiste du XVIII ${ }^{\mathrm{e}}$ siècle, dont la grande plantation, connue sous le nom d'habitation aux Isles d'Amérique, était l'unité de base. Véritable creuset de l'ensemble des sociétés créoles antillaises, qu'elles soient francophones, anglophones, ou hispanophones, l'habitation - sucrerie, caféière, indigoterie, cotonnerie..., ou autres - fut, en effet, le cadre de vie, de mort et de travail quotidien de la majorité des esclaves - nègres à talents, le plus souvent créoles (nés sur place), ou nègres de houe, ou de jardin, en grande majorité bossales (transportés d'Afrique). Elle a perduré longtemps après les abolitions de l'esclavage. Entre 1975 et 1990, à partir de repérages effectués dans des documents d'archives des XVIII ${ }^{\mathrm{e}}$ et XIX ${ }^{\mathrm{e}}$ siècles suivis d'explorations et d'enquêtes d'archéologie industrielle de terrain, plus de 2500 clichés de vestiges de la société d'habitation coloniale esclavagiste de Saint-Domingue et de son environnement urbain, militaire, religieux ou naturel, ont été pris en Haïti afin de garder trace d'un patrimoine menacé. Depuis 2009, avec l'aide du CNRS, le CIRESC a entrepris la mise en ligne d'une sélection d'environ 500 de ces documents du fonds Jacques de Cauna qui sont présentés pour la première fois à l'occasion de ce colloque, répondant ainsi en partie au vœu formulé à l'époque par ses étudiants haïtiens : Les sucreries sont la sueur et le sang de nos aïeux: il faut les respecter.

Haïti, first black republic in the world, is nowadays a true conservatory of the historical heritage of the eighteenth century slavery economics. The system was in fact based on the primary unit of the large-scale plantation, known in the French islands of America as habitation. Even in French, English or Spanish speaking Caribbean, it is there - on sugar estates, coffee, indigo or cotton plantations, or any other "places" - that lie the foundations of all creole societies, as well as the frame of everyday life, work and death, of the best part of the slaves - were they domestic or specialized workers, most of them creols (caribbean natives), or simple field workers, bossales (african born). The habitation system remained a long time after the end of slavery. Between 
1975 and 1990, starting with localizations made through the help of eighteenth and nineteenth centuries archives followed by field inquiries in industrial archeology, more than 2500 photos of remains of this kind of society in ancient Saint-Domingo have been taken in Haït, along with its urban, military, religious or natural environment, in the purpose of keeping memory of an endangered heritage. Since 2009, thanks to the help of the CNRS, the CIRESC undertook to digitalize and put on line a selection of about 500 of these documents from the Jacques de Cauna fund. They are today presented for the first time. This tries to answer the wish of our Haitian students at the very start of these operations: "Sugar estates are the sweat and blood of our ancestors. We have to show them respect".

INDEX

Mots-clés : patrimoine, mémoire, vestiges archéologiques, esclavage, habitations, sucreries, Saint-Domingue/Haïti

Keywords : heritage, memory, archéological remains, slavery, plantations, sugar-estates, Haïti/ Saint-Domingo.

\section{AUTEUR}

\section{JACQUES DE CAUNA}

Docteur d'État habilité de la Sorbonne, Membre du Conseil scientifique du CIRESC, jdecauna40@aol.com 